\title{
Berberine inhibits enterovirus 71 replication by downregulating the MEK/ERK signaling pathway and autophagy
}

\author{
Huiqiang Wang ${ }^{1}$, Ke Li ${ }^{1}$, Linlin Ma', Shuo $\mathrm{Wu}^{1}$, Jin Hu${ }^{1}$, Haiyan Yan', Jiandong Jiang ${ }^{1,2}$ and Yuhuan $\mathrm{Li}^{1 *}$
}

\begin{abstract}
Background: The MEK-ERK signaling pathway and autophagy play an important role for enterovirus71(EV71) replication. Inhibition of MEK-ERK signaling pathway and autophagy is shown to impair EV71 replication. Berberine (BBR), an isoquinoline alkaloid isolated from Berberis vulgaris L., has been reported to have ability to regulate this signaling pathway and autophagy. Herein, we want to determine whether berberine can inhibit EV71 infection by downregulating the MEK/ERK signaling pathway and autophagy.
\end{abstract}

Methods: The antiviral effect of berberine was determined by cytopathic effect (CPE) assay, western blotting assay and qRT-PCR assay. The mechanism of BBR anti-virus was determined by western blotting assay and immunofluorescence assay.

Results: We showed that berberine does-dependently reduced EV71 RNA and protein synthesis, which was, at least in part, the result of inhibition of activation of MEK/ERK signaling pathway. Furthermore, we found that berberine suppressed the EV71-induced autophagy by activating AKT protein and inhibiting the phosphorylation of JNK and PI3KIII.

Conclusions: BBR inhibited EV71 replication by downregulating autophagy and MEK/ERK signaling pathway. These findings suggest that BBR may be a potential agent or supplement against EV71 infection.

Keywords: Enterovirus 71 (EV71), Berberine, Antiviral activity, Autophagy, MEK/ERK signaling pathway

\section{Background}

Enterovirus, which belongs to the Picornaviridae family, is often associated with a serious infectious disease affecting millions of people worldwide. In particular, Enterovirus 71 (EV71) is the most common cause for hand, foot and mouth disease (HFMD) in children under the age of five [1]. EV71 was first isolated from patients in California in 1969. Since then, its outbreaks have been periodically reported worldwide, especially in the Asia-Pacific region. EV71-caused HFMD is often associated with severe neurological diseases and fatalities. Unfortunately, currently there are no effective antiviral drugs in the clinic to treat EV71-induced HFMD [2, 3].

\footnotetext{
*Correspondence: yuhuanlibj@126.com

${ }^{1}$ Institute of Medicinal Biotechnology, Chinese Academy of Medical Sciences and Peking Union Medical College, Beijing 100050, China

Full list of author information is available at the end of the article
}

It is known that viruses successfully infect host cells, needing toutilizemany functional components of different cellular signaling pathways in cells. Mitogen activated protein kinases (MAPKs) are important molecules mediating innate immunity in viral infection and activation of the MEK/ERK MAPK signaling pathway has been shown to be essential for EV71 replication in embryonic rhabdomyosarcoma (RD) cells $[4,5]$, human embryonic kidney (HEK) 293 cells [4], SK-N-SH cells [6] and immature dendritic (iDCs) cells [7]. Inhibition of MEK/ERK signaling pathway by U0126 or specific siRNAs has been found to impair EV71 replication [4-7]. These recent studies strongly supports that MEK/ERK signaling pathway plays an essential role in EV71 life cycle and pathogenesis. Therefore, blockage of MEK/ERK signaling pathway may be an excellent strategy in limiting EV71 infection. 
In addition, virus-associated autophagy is also known to provide a support for viral replication. Much evidence shows that viral infection induces autophagy in host cells including human cytomegalovirus [8], hepatitis $C$ virus [9], herpes simplex virus type I [10], coxsackievirus B3 [11], influenza A virus [12], human immunodeficiency virus type I [13], and EV71 [14-16]. Autophagy induced by viruses infection may provide a support for viruses replication. It has been reported that EV71 infection could induce autophagic machinery to promote EV71 replication in vivo and in vitro [14]. Fu et al. demonstrated that EV71 infection African green monkey kindy cells (Vero) induced autophagy and they identified a miRNA, miR-30a, that inhibited EV71 replication by modulating EV71-induced autophagy [16]. Lee et al. found that EV71-induced autophagy increases viral replication and pathogenesis in a suckling mouse model [17]. Therefore, therapeutic modulation of autophagy may be a promising strategy for inhibiting EV71 replication.

Berberine (BBR, Fig. 1a), an isoquinoline alkaloid isolated from Berberis vulgaris L., has been reported to have multi pharmacological effects such as antidiarrheal effect [18], antibacterial effect [19], hypotensive effect [20], and antiviral activity [21, 22]. Most of these pharmacological effects is known to be associated with the mechanisms that BBR regulates signaling pathway in vitro, such as, EGFR/MEK/ERK signaling pathway [23], NF- $\mathrm{KB}$ pathway [22], AMPK/mTOR signaling pathway
[24]. In addition, Deng et al. demonstrated that BBR attenuates autophagy in adipocytes by targeting BECN1 [25]. These findings suggest that BBR can regulate MEK/ERK signaling pathway and autophagy, which implies that BBR might be effective in limiting viral replication. In line with this notion, BBR is shown to inhibit the replication of respiratory syncytial virus (RSV), herpes simplex virus (HSV), human papilomavirus (HPV), and human cytomegalovirus (HCMV) [21, 22, 26, 27]. However, it is unclear that BBR has an inhibitory action against EV71. Herein, the aim of our research is to determine whether BBR inhibits EV71 replication by downregulating autophagy and MEK/ERK signaling pathway.

\section{Results}

BBR inhibits EV71 replication

To determine the anti-EV71 activities of the tested compounds, their abilities to inhibit EV71-induced CPE in Vero cells. First, we first detected the toxicity of BBR and pirodavir in Vero cells by Cell Counting Kit (CCK) assay (Fig.1c, d). As shown in Table 1, the $50 \%$ toxicity concentration $\left(\mathrm{TC}_{50}\right.$ ) of $\mathrm{BBR}$ was $73.10 \mu \mathrm{mol} / \mathrm{L}$ in Vero cells and the $\mathrm{TC}_{50}$ of pirodavir (Fig. 1b) was $27.49 \mu \mathrm{mol} / \mathrm{L}$ in Vero cells.

Next,we performed CPE assays according to the above results. We found that BBR significantly inhibited the replication of all the tested strains, including H, JS-52,
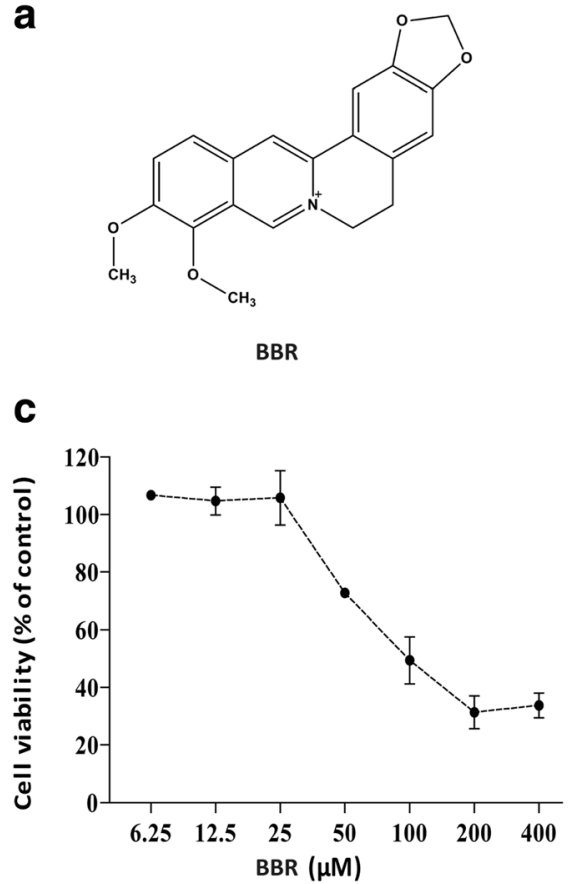

b<smiles>CCOC(=O)c1ccc(OCCC2CCN(c3ccc(C)nn3)CC2)cc1</smiles>

pirodavir

d

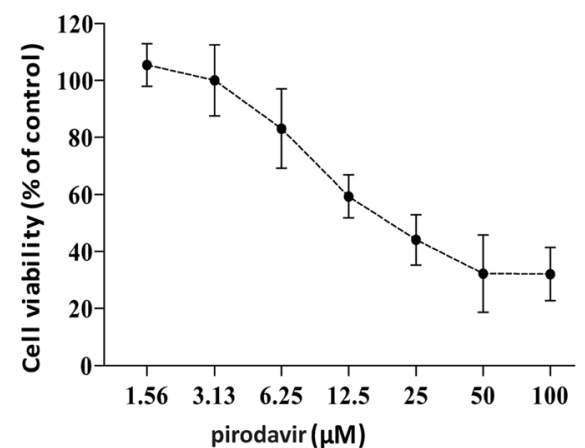

Fig. 1 The chemical structure of compounds and CCK assay. a The chemical structure of BBR. $\mathbf{b}$ The chemical structure of pirodavir. $\mathbf{c}$ The CCK assay of BBR. $\mathbf{d}$ The CCK assay of pirodavir 
Table 1 The efficiency of BBR and pirodavir against EV71 in vitro

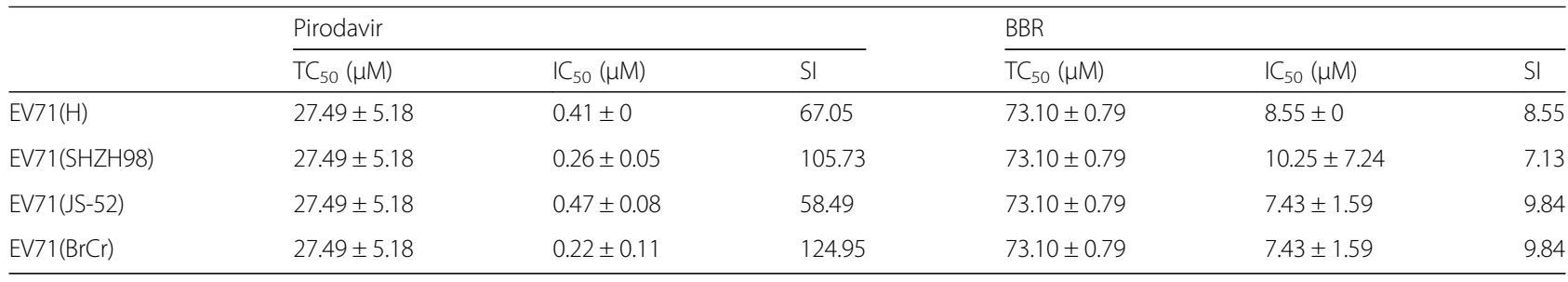

Values provided in this table represent the mean of three independent experiments

SHZH98 and BrCr stains, with the 50\% inhibitory concentration $\left(\mathrm{IC}_{50}\right)$ values ranging from 7.43 to $10.25 \mu \mathrm{M}$ (Table 1).

Similarly, the activity of BBR anti-EV71 virus also visually demonstrated with electron microscope photograph and crystal violet staining in Vero cells (Fig.2a and b). To futher confirm the inhibitory action of BBR and pirodavir against EV71, the expression of VP1 and EV71 capsid protein, was analyzed to examine its effect on EV71 biological synthesis. As shown in Fig. 2d, BBR decreased the expression of VP1 protein in a dose dependent manner. In the same way, BBR treatment dose-dependently decreased the level of VP1 RNA measured by reverse transcription-quantitative polymerase chain reaction (RT-qPCR) assay (Fig. 2c). The reference drug Pirodavir also significantly decreased the RNA and protein synthesis of EV71. Collectively, BBR demonstrated a potent inhibitory activity against EV71 virus.
BBR inhibits the phosphorylation of MEK/ERK signaling pathway

It was known that MEK/ERK were downstream components of epidermal growth factor receptor (EGFR) signaling pathways which can be activated by several stimulus including EV71 infection in various cell types $[6,28]$. Inhibition of MEK/ERK signaling pathway by U0126 or specific siRNAs has been found to impair EV71 replication [4-7] and BBR was reported to regulate this signaling pathway in vitro. Thus, we assessed whether BBR affected EV71 replication by regulating the phosphorylation of MEK/ERK. As shown in Fig.3, both BBR and poridavir inhibited EV71 replication. However, the phosphorylation of MEK/ERK was significantly attenuated by treatment with BBR but not pirodavir.

\section{BBR inhibits EV71-induced autophagy}

Autophagy induced by virus infection provides a support for viral replication. It has been reported that EV71
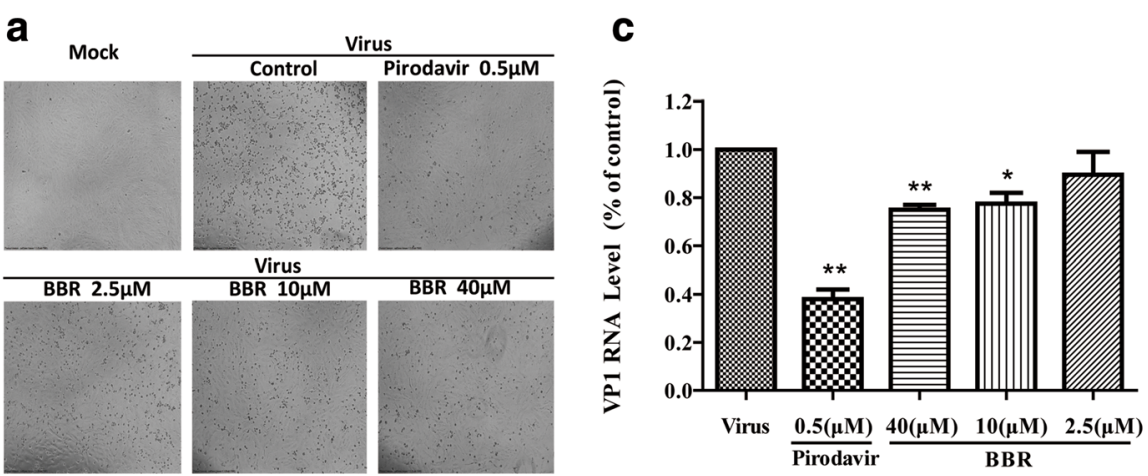

b

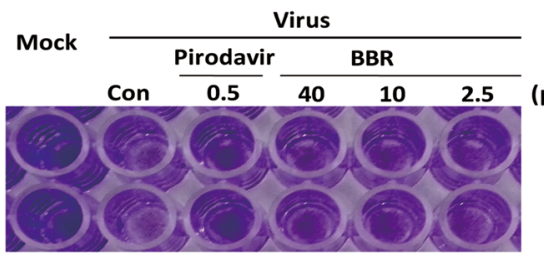

d

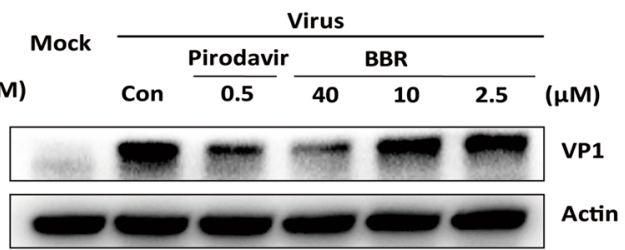

Fig. 2 The antiviral effect of BBR and pirodavir against EV71 in Vero. a BBR and pirodavir reduced the EV71-induced CPE in Vero cells. Cells were examined using a microscopy $(\times 40)$. b BBR and pirodavir reduced the EV71-induced CPE in Vero cells. Cells were examined using crystal violet staining. $\mathbf{c} B B R$ and pirodavir reduced the expression of EV71 VP1 RNA by one-step qRT-PCR assay. ${ }^{*} P<0.001{ }^{*} P<0.05 \mathbf{d}$ BBR and pirodavir reduced the expression of EV71 VP1 protein in Vero cells by western blot assay 


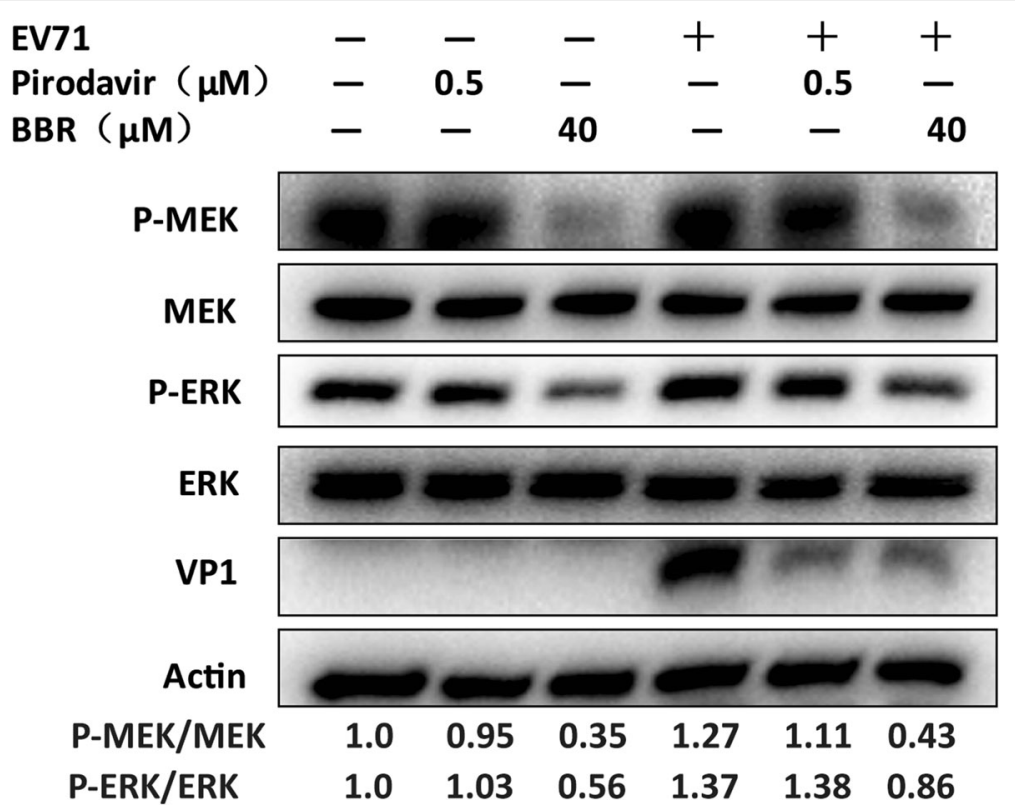

Fig. 3 BBR but not pirodavir reduces the phosphorylation of MEK/ERK. Vero cells $\left(9 \times 10^{5}\right.$ cells/well) were plated into 6-well culture plates. Vero cells were mock-infected or infected with $\mathrm{EV71}(\mathrm{H}, \mathrm{MOI}=0.1)$ for $1 \mathrm{~h}$. The cells were then treated with BBR $(40 \mu \mathrm{mol} / \mathrm{L})$ and pirodavir $(0.5 \mu \mathrm{mol} / \mathrm{L})$, respectively, for $24 \mathrm{~h}$. The cells were harvested and proteins were examined by western blot

infection could induce autophagic machinery to promote EV71 replication in vivo and in vitro [14]. JNK signaling pathway plays an important role in regulation of cell growth, proliferation, differentiation, migration and apoptosis. Studies have shown that JNK signaling pathway is closely related to autophagy, and inhibition of JNK signaling pathway could inhibit autophagy. PI3KIII, as an upstream activator of autophagy, plays an important role in autophagy.

Otherwise, AKT, as an upstream regulator of autophagy, is usually considered to be an autophagy inhibitor. We speculated that BBR inhibited EV71-induced autophagy by affecting JNK, PI3KIII and AKT signaling pathway. As shown in Fig.4a, BBR increased AKT
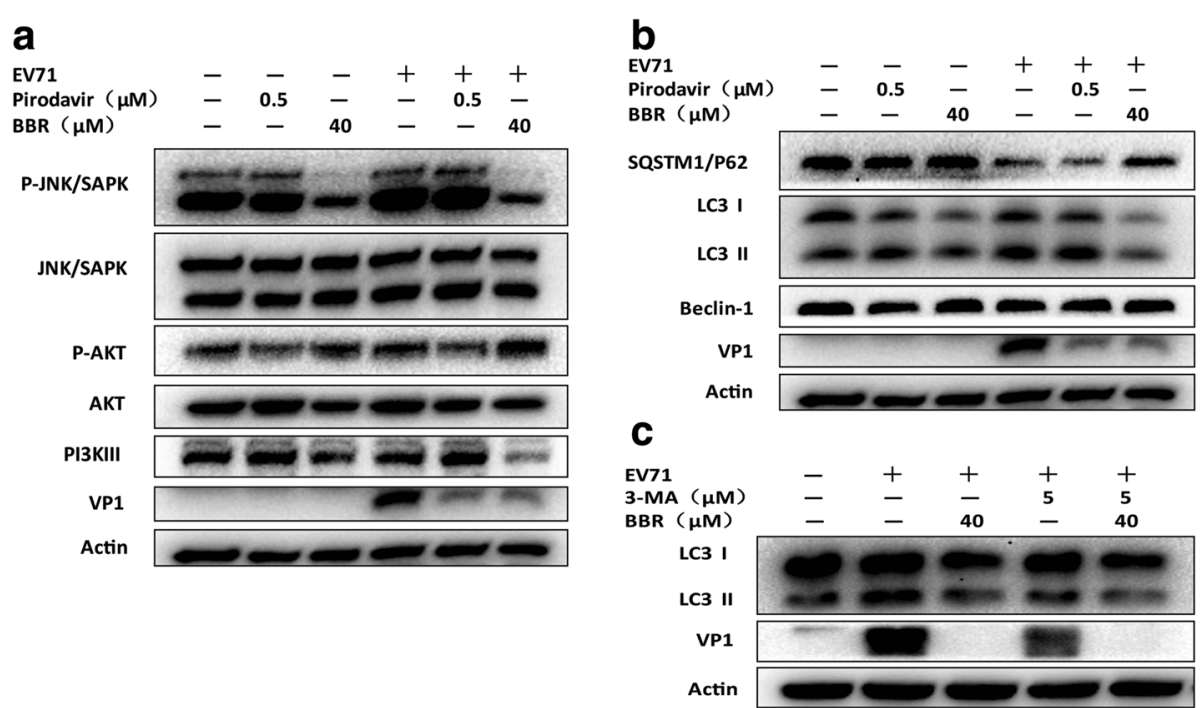

Fig. 4 BBR could inhibit EV71-induced autophagy. (a, b) BBR but not pirodavir can reduce EV71-induced autophagy. Vero cells were mock-infected or infected with EV71 $(\mathrm{H}, \mathrm{MOl}=0.1)$ for $1 \mathrm{~h}$. The cells were then treated with BBR $(40 \mu \mathrm{mol} / \mathrm{L})$ and pirodavir $(0.5 \mu \mathrm{mol} / \mathrm{L})$, respectively, for $24 \mathrm{~h}$. The cells were harvested and proteins were examined by western blot. (c) BBR and 3-MA could both reduce EV71 infection by inhibiting autophagy. Vero cells were mock-infected or infected with EV71 $(\mathrm{H}, \mathrm{MOI}=0.1)$ for $1 \mathrm{~h}$. The cells were then treated with BBR $(40 \mu \mathrm{mol} / \mathrm{L})$ and $3-\mathrm{MA}(5 \mu \mathrm{mol} / \mathrm{L})$, respectively, for $24 \mathrm{~h}$. The cells were harvested and proteins were examined by western blot 
phosphorylation and reduce JNK and PI3KIII phosphorylation. Accordingly, we found that the lapidated LC3BII, a marker for autophagy, decreased in the presence of BBR. Consistent with this, the amount of SQSTM1/P62 increased in BBR-treated cells compared to virus control, which suggested that BBR was effective in inhibiting autophagy. However, BECN-1 expression was not affected by BBR (Fig.4b). To further confirm the result of BBR inhibition of autophagy, we next used fluorescence microscopy to observe the effect of BBR on EV71-induce autophagy. As shown in Fig.5, LC3B expression was inhibited by BBR not pirodavir in EV71infected Vero cells. Similarly, 3-MA, an autophagy suppressor, could also inhibit EV71 replication (Fig.4c). Therefore, EV71-induced autophagy in Vero cells was attenuated by BBR but not pirodavir although both two compounds could inhibit EV71 replication.

\section{Discussion}

EV71, a member of the Picornaviridae family, causes HFMD by spreading through contact with viruscontaining body fluids, respiratory droplets, and feces.
However, there are no effective antiviral drugs available for the treatment of HFMD. Some natural medicinal compounds have been demonstrated to be active against the disease by ameliorating the symptoms and shortening the course $[29,30]$. Three capsid-targeting molecules known as "WIN" compounds are currently in clinical development [31, 32]. According to our results (date not shown), the antiviral activity of pirodavir against EV71 is better than pleconaril. Thus, we used pirodavir as positive control for the evaluation of the antiviral activity of BBR.

BBR has been reported to have multi pharmacological effects such as antibacterial effect [19], hypotensive effect [33], and antiviral activity [21, 22]. It has been reported that BBR could regulate signaling pathway in vitro, such as, EGFR/MEK/ERK signaling pathway [23], AMPK/mTOR signaling pathway [24]. It is interesting thing that BBR should promote EV71 replication since BBR activated ERK, which is required for the viral replication. However, BBR exhibited inhibited activity against EV71 in a dose-dependent manner. BBR inhibited the activation MEK/ERK signaling pathway in Vero cells.

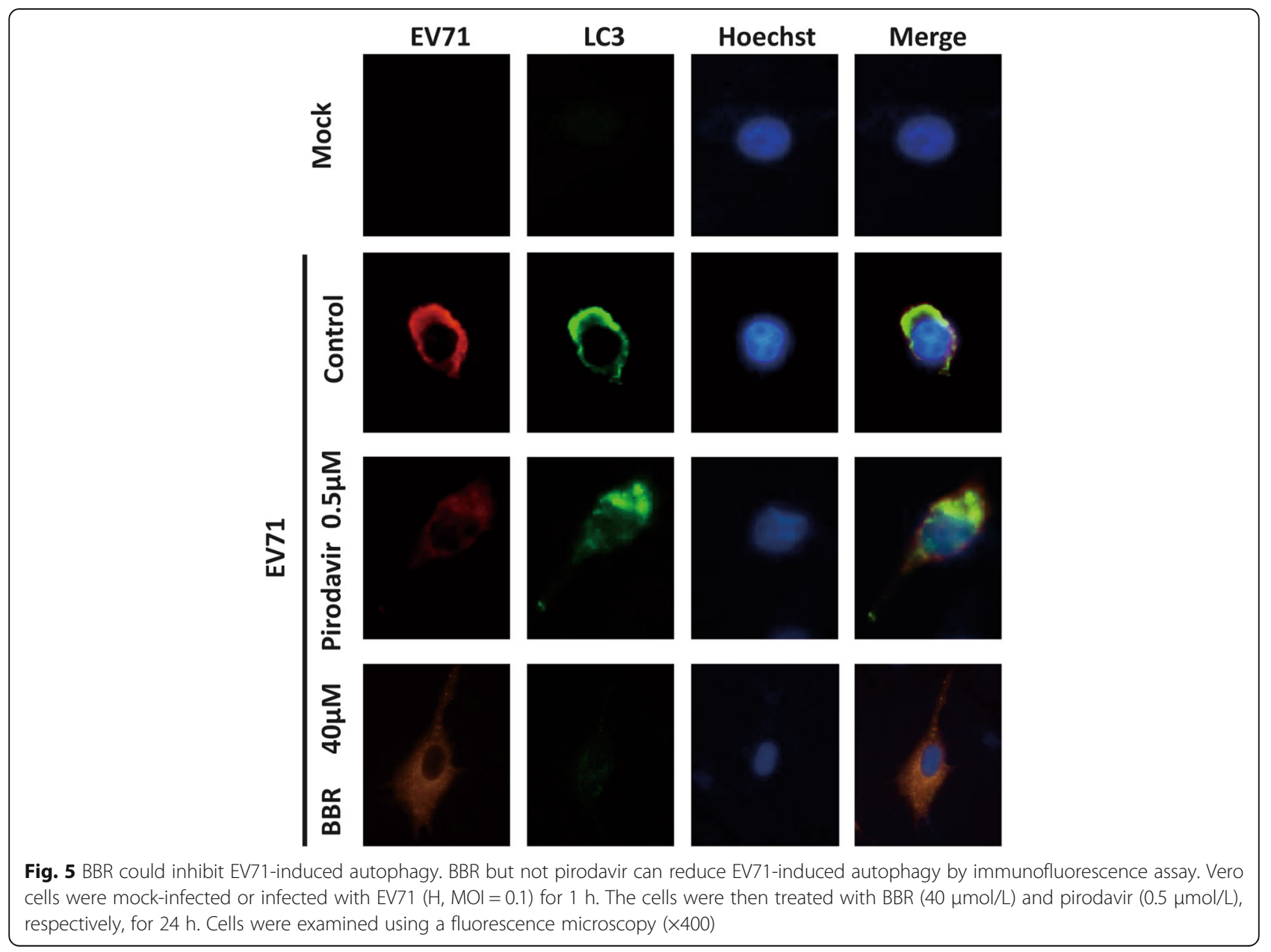


Also, Liu et al. found that BBR induced senescence of human glioblastoma cells by downregulating the EGFR/ MEK/ERK signaling pathway [23]. We thus think that BBR may have different effects on the same signaling pathway in different cell lines.

Otherwise, the preliminary analysis indicated that BBR inhibited EV71-induced autophagy. BBR suppressed LC3BII in both normal Vero cells and EV71-infected Vero cells. These findings suggest that BBR inhibits EV71 replication,at least partly by inhibiting autophagy. Of note, several studies showed that BBR also upregulated autophagy in some cell lines such as J774A.1 cells [24] and HepG2 cells [34]. Obviously, it is very possible that BBR has different effect on autophagy in different cell lines.

Overall, our research is the first time to report the anti-EV71 mechanism of BBR and our findings provide a new clue for developing the anti-EV71 drug by inhibiting MEK/ERK signaling and EV71-induced autophagy. However, many questions remain to be solved, e.g., whether berberine is effective in limiting EV71 infection in vivo. In future, we would further explore these questions in mouse models prior to clinical trials in further studies.

\section{Conclusions}

In this study, we found that BBR inhibited EV71induced autophagy and the activation of MEK/ERK signaling pathway. These findings suggest that berberine might be a potential lead or supplement for the development of new anti-EV71 agent in the future.

\section{Methods}

\section{Cells and viruses}

African green monkey kidney (Vero) cells were purchased from the American Type Culture Collection (ATCC), and were cultured in Minimum Essential Medium (MEM) supplemented with 10\% fetal bovine serum (FBS) (GIBCO) and antibiotics $(100 \mathrm{U} / \mathrm{ml}$ penicillin and $100 \mathrm{mg} / \mathrm{ml}$ streptomycin) at $37{ }^{\circ} \mathrm{C}$ in a $5 \% \mathrm{CO}_{2}$ incubator.

EV71 strain SHZH98 isolated from the throat swab sample of an HFMD case occurring in 1998 in China was kindly provided by Dr. Qi Jin, Institute of Pathogen Biology, Chinese Academy of Medical Science and Peking Union Medical School, Beijing, China. EV71 strain BrCr (VR1775) and H (VR-1432) were purchased from the ATCC. EV71 strain JS-52 was a kind gift from Dr. Xiangzhong Ye, Beijing Wantai Biological Pharmacy Enterprise Co., Ltd. EV71 were all passaged in Vero cells.

\section{Compounds}

BBR was purchased from the Sigma-Aldrich (St Louis, MO, USA) and the purity is no less than $99 \%$. Pirodavir was purchased from Biochempartner (Shanghai, China). Both BBR and piradavir were dissolved into DMSO.

\section{Cytotoxicity assay}

Cytotoxic effects of BBR on Vero cells were assayed by CCK (TransGen Biotech, China) assay. Briefly, cells $\left(3 \times 10^{4}\right.$ cells/well $)$ were seeded into 96-well culture plates and were incubated overnight. Then, the medium was removed and different concentrations of BBR were applied in triplicate. After 3 days' incubation, the cytotoxicity of BBR was determined by CCK assay. The signals were read at $450 \mathrm{~nm}$ on Enspire (Perkin Elmer,Waltham, MA, USA). The $\mathrm{TC}_{50}$ was defined as the concentration that inhibits 50\% cellular growth in comparison with the controls.

\section{CPE inhibition assay for anti-EV71}

The anti-EV71 activity of BBR was assayed by CPE inhibition method. Briefly, cells $\left(3 \times 10^{4}\right.$ cells/well $)$ were plated into 96-well culture plates for incubation of $24 \mathrm{~h}$. The medium was then removed and cells were infected with EV71 of $100 \mathrm{TCID}_{50}$ (50\% tissue culture infective doses) in serum-free medium for $1 \mathrm{~h}$ at $37^{\circ} \mathrm{C}$. Then, the unbound viruses were removed and various concentrations of BBR were supplemented for incubation of another $48 \mathrm{~h}$. The $\mathrm{IC}_{50}$ defined as the minimal concentration of inhibitor required to inhibit $50 \%$ of CPE was determined by Reed \& Muench method. The selectivity index (SI) was calculated as the ratio of $\mathrm{TC}_{50} / \mathrm{IC}_{50}$. In addition, the cells were stained with $0.5 \%$ crystal violet in $20 \%$ ethanol for $15 \mathrm{~min}$ at room temperature and the cells were imaged after rinsed with PBS.

\section{Western blot analysis}

The cells were lysed in the M-PER mammalian protein extraction reagent (Thermo, Rockford, IL) containing halt protease inhibitor single-use cocktail (Thermo). The protein concentration was determined by the $\mathrm{BCA}$ reagents (Thermo). About $15 \mu \mathrm{g}$ proteins were denatured and applied to sodium dodecyl sulfate-polyacrylamide gel electrophoresis (SDS-PAGE). The electrophoresis products were transferred to a polyvinyl idenefluoride (PVDF) film and PVDF membranes were then incubated at room temperature with specific primary antibody. After a standard washing, membranes were incubated with horse radish peroxidase (HRP)-labeled secondary antibody. The assay developed using a chemiluminescent substrate. The primary antibodies used in this study included antibodies against $\beta$-actin, p-p44/p42 MAPK, p44/p42 MAPK, p-MEK, MEK, p-AKT, AKT, p-JNK, JNK, PI3KIII, SQSTM1/P62, LC3B, Beclin-1 (Cell Signaling Technology), and EV71-VP1 (Abnova). The goat anti-rabbit and anti-mouse HRP-labeled antibodies were obtained from Cell Signaling Technology. 


\section{Immunofluorescence assay}

Vero cells grown on glass coverslips (Thermo) were infected with EV71 $(\mathrm{H}, \mathrm{MOI}=0.1)$ for $1 \mathrm{~h}$. Then, BBR and pirodavir were supplemented for incubation of another $24 \mathrm{~h}$. After incubation, the culture medium was removed and the cells were washed and fixed. The cells were permeabilized in $0.5 \%$ Triton X-100 at room temperature for $15 \mathrm{~min}$ and blocked in PBS containing 1\% BSA for $60 \mathrm{~min}$ at room temperature. Cells were then incubated with an anti-EV71 antibody (Millipore) and LC3B (Cell Signaling Technology) antibody at a dilution of 1: 500 for $2 \mathrm{~h}$ at room temperature. After washing three times with PBS, the samples were reacted with PE conjugate goat anti-mouse secondary antibody (TransGen Biotech, China) and FITC conjugate goat anti-rabbit secondary antibody (TransGen Biotech, China) at a dilution of 1: 500 for $1 \mathrm{~h}$ at room temperature. After washing with PBS, add Hoechst (Beyotime Institute of Biotechnology, China) for $10 \mathrm{~min}$ and images were taken using a fluorescence microscope (Olympus, IX71).

\section{Quantitative reverse-transcription polymerase chain reaction (qRT-PCR) quantification}

Vero cells $\left(9 \times 10^{5}\right.$ cells/well $)$ were plated into 6-well culture plates for incubation of $16 \mathrm{~h}$. The medium was removed and cells were infected with EV71 $(\mathrm{H}, \mathrm{MOI}=$ 0.1 ). After $1 \mathrm{~h}$, various concentrations of BBR were supplemented for incubation of another $24 \mathrm{~h}$. The total RNA of the infected cells was extracted using the RNeasy Mini kit (QIAGEN) according to the manufacturer's instructions. The one-step qRT-PCR was performed with SuperScript III Platinum SYBR Green One-step RT PCR Kit (Invitrogen) using the ABI 7500 Fast Real-Time PCR system (Applied Biosystems). The mRNA expression of EV71 VP1 was detected with sense primer 5' - GATATCCCACATTCGGTGA -3' and antisense primer $5^{\prime}$ - TAGGACACGCTCCATACTCAAG $-3^{\prime}$ targeting a conserved region of the VP1 gene. The $\beta$ actin mRNA was detected using sense primers $5^{\prime}$-TG ACGGGGT CACCCACA CTGTGCCCATCTA-3' and antisense primer 5'-CTAGAAGCATTTG CGGTGGAC G ATG-3'. PCR assay was carried out in a $25 \mu \mathrm{L}$ volume and the target fragment amplification was carried out as follows: reverse transcription at $50{ }^{\circ} \mathrm{C}$ for $3 \mathrm{~min}$; initial activation of HotStar Taq DNA Polymerase at $95^{\circ} \mathrm{C}$ for $10 \mathrm{~min} ; 40$ cycles in two steps: $95^{\circ} \mathrm{C}$ for $15 \mathrm{~s}, 60{ }^{\circ} \mathrm{C}$ for 30s. The relative amounts of EV71 VP1 mRNA was calculated by comparative $\mathrm{Ct}$ method after normalizing the quantity of $\beta$-actin.

\section{Statistical analysis}

Data are expressed as the mean \pm standard error of the mean and analyzed using two-tailed Student's $t$-tests with $P<0.001$ and $P<0.05$ taken as significant.

\section{Abbreviations}

BBR: Berberine; CNS: Central nervous system; CPE: Cytopathic effect; EV71: Enterovirus 71; HFMD: Hand foot and mouth disease; $I_{50}$ : 50\% inhibitory concentration; SI: Selectivity index; $\mathrm{TC}_{50}: 50 \%$ toxicity concentration

\section{Acknowledgments}

We are grateful to Dr. Qi Jin, Institute of Pathogen Biology, Chinese Academy of Medical Science and Peking Union Medical School (Beijing, China) for providing EV71 strain SHZH98. We are also grateful to Dr. Xiangzhong Ye, Beijing Wantai Biological Pharmacy Enterprise Co., Ltd for providing EV71 strain JS52.

\section{Funding}

The work was supported by the National Natural Science Foundation of China (Grant 81503118 and 81274101) and the Science Fund for Creative Re- search Groups of the National Natural Science Foundation of China (Grant 81321004). The work was also supported by the National Science and Technology Major Project of the Ministry of Science and Technology of China (2012ZX09301002-001-015) and CAMS Initiative for Innovative Medicine (CAMS-12M-1-010).

\section{Availability of data and materials}

The datasets supporting the conclusions of this article are included within the article.

\section{Authors' contributions}

HQ Wang and K Li designed and performed all the experiments and drafted the manuscript. LL Ma provided suggestions on the experimental design and helped edit the manuscript. S Wu performed the experiment of viral infection. J Hu and HY Yan provides advice in this study. JD Jiang and YH Li designed this study, analyzed experimental data and edited this manuscript. All authors read and approved the final manuscript.

\section{Competing interests}

The authors have declare that they have no competing interests.

\section{Consent for publication}

Not applicable.

\section{Ethics approval and consent to participate}

Not applicable.

\section{Author details}

${ }^{1}$ Institute of Medicinal Biotechnology, Chinese Academy of Medical Sciences and Peking Union Medical College, Beijing 100050, China. ${ }^{2}$ Institute of Materia Medica, Chinese Academy of Medical Sciences and Peking Union Medical College, Beijing 100050, China.

Received: 26 August 2016 Accepted: 26 December 2016

Published online: 11 January 2017

\section{References}

1. Wang HQ, Meng S, Li ZR, Peng ZG, Han YX, Guo SS, Cui XL, Li YH, Jiang JD. The antiviral effect of 7-hydroxyisoflavone against enterovirus 71 in vitro. J Asian Nat Prod Res. 2013;15(4):382-9.

2. McMinn PC. Recent advances in the molecular epidemiology and control of human enterovirus 71 infection. Curr Opin Virol. 2012;2(2):199-205.

3. Wang X, Zhu C, Bao W, Zhao K, Niu J, Yu XF, Zhang W. Characterization of full-length enterovirus 71 strains from severe and mild disease patients in northeastern China. PLoS One. 2012;7(3):e32405.

4. Wang B, Zhang H, Zhu M, Luo Z, Peng Y. MEK1-ERKs signal cascade is required for the replication of Enterovirus 71 (EV71). Antiviral Res. 2012; 93(1):110-7.

5. Zhu M, Duan H, Gao M, Zhang H, Peng Y. Both ERK1 and ERK2 are required for enterovirus 71 (EV71) efficient replication. Viruses. 2015;7(3):1344-56.

6. Tung $\mathrm{WH}$, Hsieh $\mathrm{HL}$, Yang CM. Enterovirus 71 induces COX-2 expression via MAPKs, NF-kappaB, and AP-1 in SK-N-SH cells: Role of PGE(2) in viral replication. Cell Signal. 2010;22(2):234-46.

7. Shi W, Hou X, Peng H, Zhang L, Li Y, Gu Z, Jiang Q, Shi M, Ji Y, Jiang J. MEK ERK signaling pathway is required for enterovirus 71 replication in immature dendritic cells. Virol J. 2014;11:227. 
8. Mouna L, Hernandez E, Bonte D, Brost R, Amazit L, Delgui LR, Brune W, Geballe AP, Beau I, Esclatine A. Analysis of the role of autophagy inhibition by two complementary human cytomegalovirus BECN1/Beclin 1-binding proteins. Autophagy. 2016;12(2):327-42.

9. Liu C, Qu A, Han X, Wang Y. HCV core protein represses the apoptosis and improves the autophagy of human hepatocytes. Int J Clin Exp Med. 2015; 8(9):15787-93.

10. Talloczy Z, Jiang W, Virgin HW, Leib DA, Scheuner D, Kaufman RJ, Eskelinen EL, Levine B. Regulation of starvation- and virus-induced autophagy by the elF2alpha kinase signaling pathway. Proc Natl Acad Sci U S A. 2002;99(1):190-5.

11. Wong J, Zhang J, Si X, Gao G, Mao I, McManus BM, Luo H. Autophagosome supports coxsackievirus B3 replication in host cells. J Virol. 2008;82(18):9143-53.

12. Zhou Z, Jiang X, Liu D, Fan Z, Hu X, Yan J, Wang M, Gao GF. Autophagy is involved in influenza a virus replication. Autophagy. 2009:5(3):321-8.

13. Espert L, Denizot M, Grimaldi M, Robert-Hebmann V, Gay B, Varbanov M, Codogno P, Biard-Piechaczyk M. Autophagy is involved in T cell death after binding of HIV-1 envelope proteins to CXCR4. J Clin Invest. 2006;116(8): 2161-72.

14. Huang SC, Chang CL, Wang PS, Tsai Y, Liu HS. Enterovirus 71-induced autophagy detected in vitro and in vivo promotes viral replication. J Med Virol. 2009;81(7):1241-52

15. Xi X, Zhang X, Wang B, Wang T, Wang J, Huang H, Jin Q, Zhao Z. The interplays between autophagy and apoptosis induced by enterovirus 71 PLoS One. 2013;8(2):e56966.

16. Fu Y, Xu W, Chen D, Feng C, Zhang L, Wang X, Lv X, Zheng N, Jin Y, Wu Z Enterovirus 71 induces autophagy by regulating has-miR-30a expression to promote viral replication. Antiviral Res. 2015:124:43-53.

17. Lee YR, Wang PS, Wang JR, Liu HS. Enterovirus 71-induced autophagy increases viral replication and pathogenesis in a suckling mouse model. J Biomed Sci. 2014;21:80

18. Rabbani GH. Mechanism and treatment of diarrhoea due to vibrio cholerae and Escherichia coli: roles of drugs and prostaglandins. Dan Med Bull. 1996; 43(2):173-85.

19. Peng L, Kang S, Yin Z, Jia R, Song X, Li L, Li Z, Zou Y, Liang X, He C, Ye G, Yin L, Shi F, LV C, Jing B. Antibacterial activity and mechanism of berberine against Streptococcus agalactiae. Int J Clin Exp Pathol. 2015;8(5):5217-23.

20. Chun YT, Yip TT, Lau KL, Kong YC, Sankawa U. A biochemical study on the hypotensive effect of berberine in rats. Gen Pharmacol. 1979;10(3):177-82.

21. Shin HB, Choi MS, Yi CM, Lee J, Kim NJ, Inn KS. Inhibition of respiratory syncytial virus replication and virus-induced p38 kinase activity by berberine. Int Immunopharmacol. 2015;27(1):65-8.

22. Song S, Qiu M, Chu Y, Chen D, Wang X, Su A, Wu Z. Downregulation of cellular c-Jun N-terminal protein kinase and NF-kappaB activation by berberine may result in inhibition of herpes simplex virus replication. Antimicrob Agents Chemother. 2014;58(9):5068-78.

23. Liu Q, Xu X, Zhao M, Wei Z, Li X, Zhang X, Liu Z, Gong Y, Shao C. Berberine induces senescence of human glioblastoma cells by downregulating the EGFR-MEK-ERK signaling pathway. Mol Cancer Ther. 2015;14(2):355-63.

24. Fan X, Wang J, Hou J, Lin C, Bensoussan A, Chang D, Liu J, Wang B. Berberine alleviates ox-LDL induced inflammatory factors by up-regulation of autophagy via AMPK/mTOR signaling pathway. J Transl Med. 2015;13:92.

25. Deng $Y, X u$ J, Zhang $X$, Yang J, Zhang D, Huang J, LV P, Shen W, Yang Y. Berberine attenuates autophagy in adipocytes by targeting BECN1. Autophagy. 2014:10(10):1776-86.

26. Mahata S, Bharti AC, Shukla S, Tyagi A, Husain SA, Das BC. Berberine modulates AP-1 activity to suppress HPV transcription and downstream signaling to induce growth arrest and apoptosis in cervical cancer cells. Mol Cancer. 2011;10:39

27. Hayashi K, Minoda K, Nagaoka Y, Hayashi T, Uesato S. Antiviral activity of berberine and related compounds against human cytomegalovirus. Bioorg Med Chem Lett. 2007;17(6):1562-4.

28. Rodemann HP, Dittmann K, Toulany M. Radiation-induced EGFR-signaling and control of DNA-damage repair. Int J Radiat Biol. 2007:83(11-12):781-91.

29. Wei Y, Fang W, Wan Z, Wang K, Yang Q, Cai X, Shi L, Yang Z. Antiviral effects against EV71 of pimprinine and its derivatives isolated from Streptomyces sp. Virol J. 2014;11:195.

30. Chen X, Wang C, Xu L, Wang W, Yang G, Tan RX, Li E, Jin Y. A laboratory evaluation of medicinal herbs used in china for the treatment of hand, foot, and mouth disease. Evid Based Complement Alternat Med. 2013;2013: 504563
31. De Clercq E. Highlights in antiviral drug research: antivirals at the horizon. Med Res Rev. 2013;33(6):1215-48.

32. Thibaut HJ, De Palma AM, Neyts J. Combating enterovirus replication: stateof-the-art on antiviral research. Biochem Pharmacol. 2012;83(2):185-92.

33. Sun T, Liu R, Cao YX. Vasorelaxant and antihypertensive effects of formononetin through endothelium-dependent and -independent mechanisms. Acta Pharmacol Sin. 2011;32(8):1009-18.

34. Yu R, Zhang ZQ, Wang B, Jiang HX, Cheng L, Shen LM. Berberine-induced apoptotic and autophagic death of HepG2 cells requires AMPK activation. Cancer Cell Int. 2014;14:19.

\section{Submit your next manuscript to BioMed Central and we will help you at every step:}

- We accept pre-submission inquiries

- Our selector tool helps you to find the most relevant journal

- We provide round the clock customer support

- Convenient online submission

- Thorough peer review

- Inclusion in PubMed and all major indexing services

- Maximum visibility for your research

Submit your manuscript at www.biomedcentral.com/submit
) Biomed Central 